\section{Krankheitsmodifizierender Effekt für 5-Gräser-Sublingualtablette gezeigt}

\begin{abstract}
A uf der Jahrestagung der American Academy of Allergy, Asthma and Immunology (AAAAI), die von 2. bis 6. März 2012 in Orlando stattgefunden hat, wurden Daten aus einer klinischen Phase-III-Studie (VO53.06) zur 5-Gräser-Tablette Oralair ${ }^{\circledR}$ vorgestellt. Bei dieser Studie handelt es sich um eine auf vier Jahre angelegte randomisierte, doppelblinde und placebokontrollierte Studie mit 633 Patienten. Drei Jahre lang wurde nach einem prä- und cosaisonalen Schema (vor und während der Pollensaison in jedem Jahr) diskontinuierlich behandelt. Im letzten Jahr wurde behandlungsfrei nachbeobachtet. Primärer Endpunkt war der durchschnittliche angepasste Symptomwert (Average Adjusted
\end{abstract}

Symptom Score, AASS). Sekundäre Wirksamkeitskriterien beinhalteten den Leidensdruck gemessen mit dem Fragebogen zur Lebensqualität mit Rhinokonjunktivitis (Rhinoconjunctivitis Quality of Life Questionnaire, RQLQ).

Die Resultate aus den vier Jahren wurden anhand dreier wissenschaftlicher Poster präsentiert [Malling $\mathrm{H}$ et al. J Allergy Clin Immunol 2012; 129: 2 Suppl. 1 (AB144) - Horak F et al. J Allergy Clin Immunol 2012; 129: 2 Suppl. 1 (AB46) Worm $\mathrm{M}$ et al. J Allergy Clin Immunol 2012; 129: 2 Suppl. 1 (AB47)]. Sie zeigten ein Jahr nach Ende der Behandlung eine fortgesetzte Wirksamkeit sowie eine klinisch signifikante Verbesserung der Le- bensqualität in jeder der vier Pollensaisonen - einschließlich der ersten Pollensaison nach der Behandlung. Die Daten zeigen außerdem, dass die sublinguale Immuntherapie bei den Patienten am besten wirkte, die an besonders schweren Formen von durch Gräserpollen induzierten Atemwegsallergien litten.

„Ich freue mich über diese Ergebnisse, da damit zum ersten Mal ein krankheitsmodifizierender Effekt einer Sublingualtablette für die Immuntherapie bei Gräserallergien im Zusammenhang mit einer diskontinuierlichen Behandlung nach prä- und cosaisonaler Anwendung festgestellt wurde“, sagte Prof. Dr. Hans-Jørgen Malling vom Nationalen Universitätskrankenhaus Kopenhagen, Dänemark, der einer der Mitverfasser der Poster ist.

Nach Informationen von Stallergenes

\section{Hilfe bei Heuschnupfen}

$\bigcup$ nterstützung bei der Beratung von Pollenallergikern erhalten Ärzte jetzt gleich aus mehreren Kanälen. Da wäre zunächst die frisch überarbeitete Website www.heuschnupfenhilfe.de: Das Kooperationsprojekt der Unternehmen Novartis (Otriven ${ }^{\circledR}$ Allergie Aktiv mit Beclometason) und SCA Hygiene Products (Tempo ${ }^{\circ}$ plus) klärt über Symptome, Ursachen und die Therapiemöglichkeiten der allergischen Rhinitis auf. Zahlreiche Tipps zum Umgang mit der Erkrankung, z. B. zur Benutzung von Kontaktlinsen, ergänzen das Angebot. Ein Highlight des Heuschnupfenportals ist eine SmartphoneApp, die über die Pollenflugbelastung der nächsten drei Tage Auskunft gibt. Die App erinnert zudem an die Einnahme von Medikamenten und meldet besonders hohe Pollenkonzentrationen. Geeignet ist das Programm für Android-Smartphones und das iPhone.

Passend dazu gibt es auch eine neue Patientenbroschüre zum Thema Heuschnupfen. Die Broschüre entstand ebenfalls in Zusammenarbeit von Novartis (Otriven ${ }^{\circledR}$ Allergie Aktiv mit Beclometason) und SCA Hygiene Products (Tempo ${ }^{\circ}$ plus). Der 14-seitige Ratgeber informiert über Entstehungsursachen und Symptome, vor allem aber über die richtige

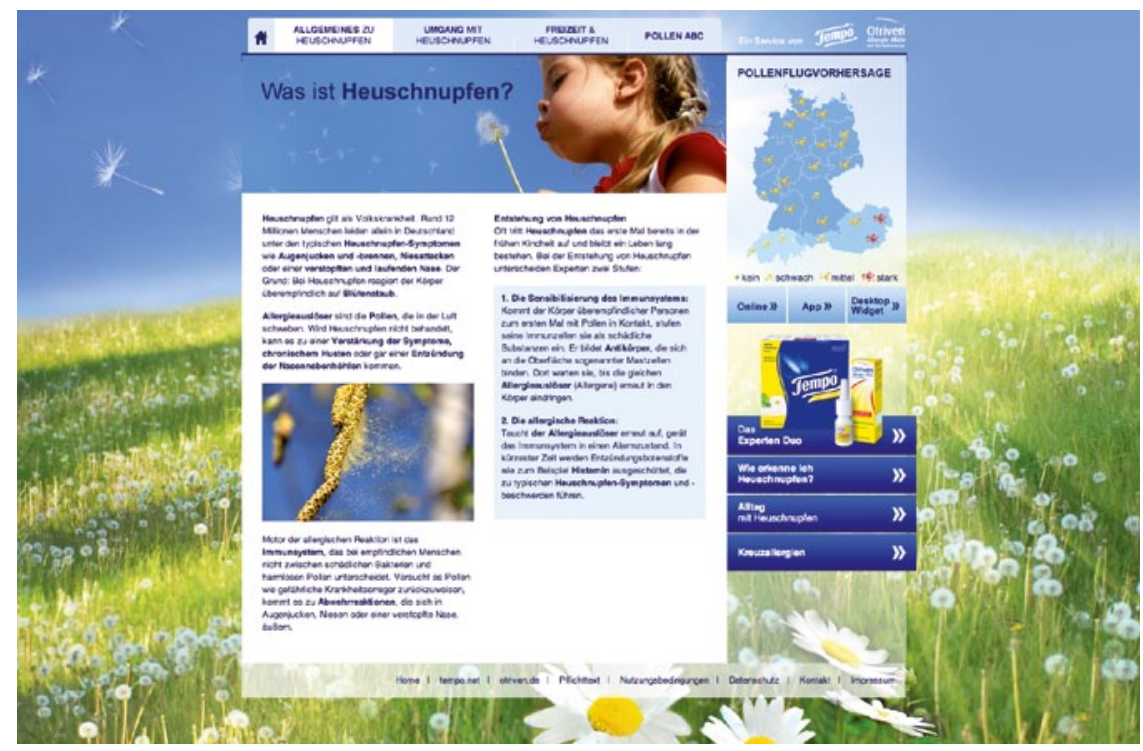

Frühlingsfrisch überarbeitet - die Website www.heuschnupfenhilfe.de kann bei der Patientenberatung hilfreich sein.

Anwendung von Nasensprays. Denn viele Betroffene wenden Nasensprays nach wie vor falsch an. Die Broschüre samt Aufsteller für die Praxis ist für Ärzte kostenlos.

Neben Website und Patientenbroschüre können Ärzte ihre Heuschnupfenpatienten nun auch noch mit einem weiteren Hilfsmittel unterstützen: Die Anwendungskarten von Otriven ${ }^{\circledR}$ Allergie Aktiv mit Beclometason veranschaulichen anhand von sechs Zeichnungen wie das
Nasenspray richtig benutzt wird. Die Karten im DIN-A6-Format eignen sich sowohl als Erklärungshilfe für Erwachsene wie auch für Kinder.

Patientenbroschüre und Anwendungskarten können Ärzte kostenlos bestellen - per Fax an (089) 78 77-3 33 oder via E-Mail an miriam.muench@edelman. com.

Nach Informationen von Novartis 